\title{
Converting MIDI Notes to ABC Notes in Pencil Code
}

By Jessie M. Heines

C omparing Scratch [4] to Pencil Code [3] for teaching computing through music immediately reveals a major difference: Scratch represents notes as MIDI numbers, while Pencil Code represents notes as letters using $\mathrm{ABC}$ notation [1]. To someone who reads music, Pencil Code is clearly preferable because it is far easier to map, for example, a major third to $\mathrm{C}$ and $\mathrm{E}$ than 60 and 64. But to those who generate music algorithmically, $\mathrm{ABC}$ is not as easy as MIDI. For example, a major third in the key of D is not D and F, it is $\mathrm{D}$ and F\#. Using MIDI values, however, the interval is always 4 semitones: 62 (D) $+4=66$ (F\#), just as before.

The Problem: Pencil Code is written in CoffeeScript [4], "a little language that compiles into JavaScript." Thus, one would think to use the String.fromCharCode function to convert numeric MIDI to alphabetic ABC. Unfortunately, the conversion is not that straightforward, because $A B C$ notation is based on a $\mathrm{C}$ scale, with $\mathrm{A}$ and $\mathrm{B}$ above $\mathrm{G}$. That throws out a simple conversion. The 4th octave, the one that begins on middle C, is represented by all capital letters: $\mathrm{C} \mathrm{D} \mathrm{E}$ F G A B. For the 5th octave, apostrophes are added: C' D' E' F' G' A' B'. For the 6th octave, two apostrophes are added, and so on to the higher octaves. For the 3rd octave, the one below middle $\mathrm{C}$, commas are added: C, D, E, F, G, A, B. And, as you can probably guess from the pattern, for the 2nd octave, two commas are added and so on to the lower octaves.

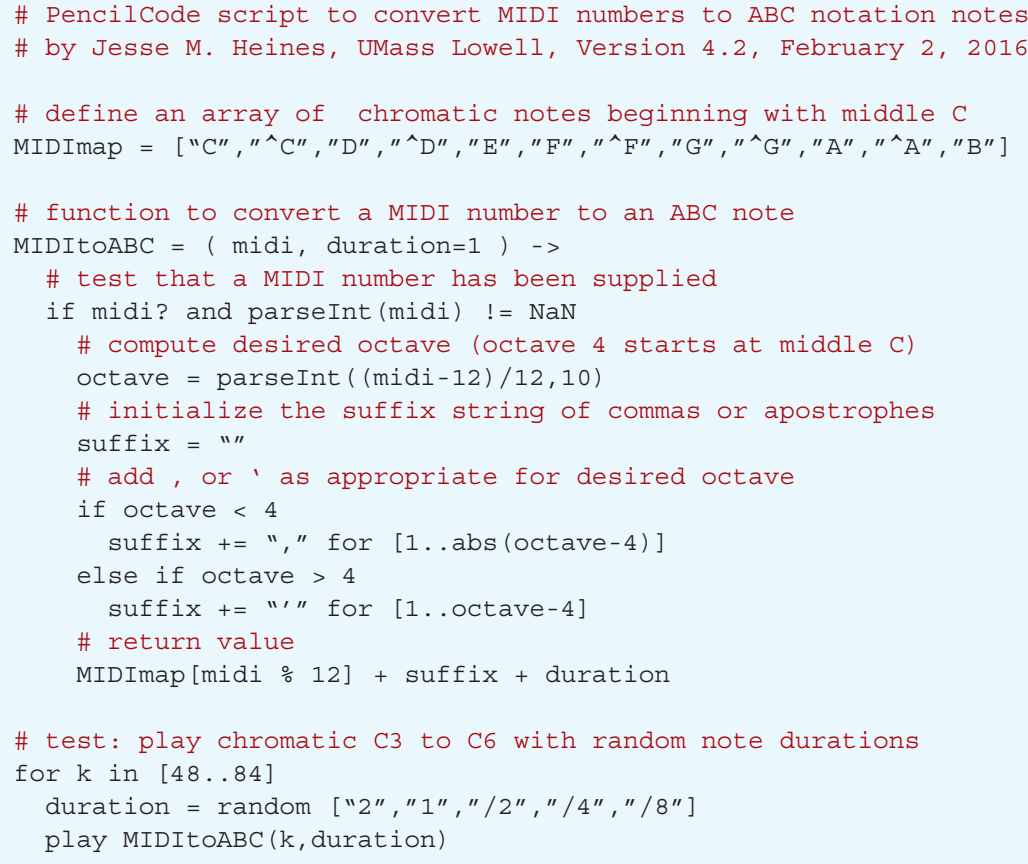

A Solution: If one wants both the clarity of $\mathrm{ABC}$ notation and the algorithmic power of MIDI values, a conversion is indeed necessary. The code below runs in Pencil Code and plays the chromatic scale from $\mathrm{C} 3$ to $\mathrm{C} 6$ by passing MIDI values 48 (C3) through 84 (C6) to a MIDItoABC function that is generalized to convert any MIDI value to its proper representation in $\mathrm{ABC}$ notation and play it for a specified duration.

A Note on ABC Duration Notation: Unrelated to MIDI but germane to ABC, duration strings are based on quarter notes, which have a duration of 1 . A half note has a duration of 2 , and a whole note a duration of 4 . Going the other way, an eighth note has a duration of $1 / 2$, designated as "/2". Similarly, a 16th note has a duration of " $/ 4$ ", and a 32nd note has a duration of " $/ 8$ ".

The code below includes a test routine to play the chromatic scale from $\mathrm{C} 3$ to $\mathrm{C} 6$ with random note durations from a half note ("2") to a 32 nd note ("/8").

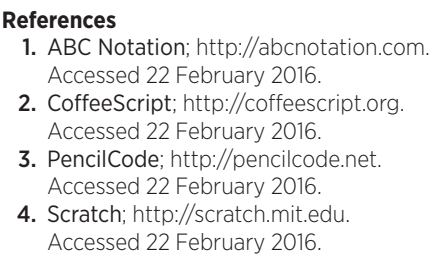

Jesse M. Heines

UMass Lowell-Computer Science

One University Avenue

Lowell, Massachusetts 01854 USA heines@cs.uml.edu 